\title{
Porin A-Specific Antibody Avidity in Patients Who Are Convalescing from Meningococcal B Disease
}

\author{
CLEMENTIEN L. VERMONT, HARRY H. VAN DIJKEN, RONALD DE GROOT, AND \\ GERMIE P.J.M. VAN DEN DOBBELSTEEN \\ Laboratory for Vaccine Research [C.L.V., H.H.v.D., G.P.J.M.v.d.D.], Netherlands Vaccine Institute, 3720 \\ AL, Bilthoven, The Netherlands; and Department of Pediatrics [C.L.V., R.d.G.], Erasmus MC-Sophia \\ Children's Hospital, 3000 CB, Rotterdam, The Netherlands
}

\begin{tabular}{|c|c|}
\hline \multicolumn{2}{|c|}{ ABSTRACT } \\
\hline 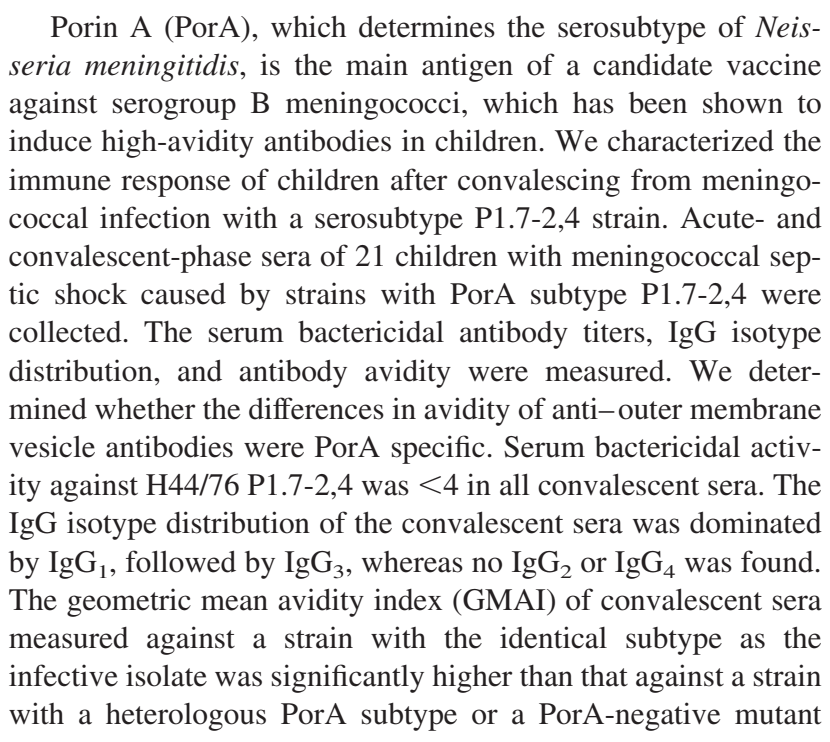 & $\begin{array}{l}\text { strain ( } 57 \text { versus } 35 \text { and } 23 \% \text {, respectively; } p=0.005 \text { and } p< \\
0.001) \text {. Geometric mean avidity titers were highest for P1.7-2,4, } \\
\text { corresponding with the highest GMAI. The GMAI after invasive } \\
\text { meningococcal disease was lower than after vaccination of } \\
\text { healthy toddlers with a monovalent P1.7-2,4 outer membrane } \\
\text { vesicle vaccine. (Pediatr Res 58: 149-152, 2005) } \\
\qquad \text { Abbreviations } \\
\text { AI, avidity index } \\
\text { CI, confidence interval } \\
\text { GMAI, geometric mean avidity index } \\
\text { GMAT, geometric mean avidity titer } \\
\text { GMT, geometric mean titer } \\
\text { LPS, lipopolysaccharide } \\
\text { NaSCN, sodium thiocyanate } \\
\text { OMV, outer membrane vesicle } \\
\text { PorA, porin A } \\
\text { PorB, porin B } \\
\text { SBA, serum bactericidal activity }\end{array}$ \\
\hline
\end{tabular}

Meningococcal meningitis and septicemia are important causes of morbidity and mortality in children, especially in those younger than $2 \mathrm{y}$. Sequelae include loss of hearing, skin necrosis, and occasionally amputation of limbs. Most cases of invasive meningococcal disease in the industrialized countries are caused by Neisseria meningitidis serogroup B. The immune response after invasive meningococcal $\mathrm{B}$ disease is thought to be protective because recurrent meningococcal $\mathrm{B}$ infections in otherwise healthy children are extremely rare. Therefore, evaluation of the immune responses after meningococcal diseases supplies important information on the magnitude and the specificity of the immune response that a protective vaccine should

Received July 7, 2004; accepted October 13, 2004

Correspondence: Germie P.J.M. van den Dobbelsteen, Ph.D., Laboratory for Vaccine Research, Netherlands Vaccine Institute, P.O. Box 457, 3720 AL Bilthoven, The Netherlands; e-mail: germie.van.den.dobbelsteen@nvi-vaccin.nl.

DOI: 10.1203/01.PDR.0000156372.13569.08 induce. IgG antibodies that are found after systemic meningococcal B infections are mainly directed against porin A (PorA), porin B (PorB), opacity protein $\mathrm{C}$, and lipopolysaccharide (LPS) (1,2). In children who were younger than $12 \mathrm{mo}$, serum bactericidal activity (SBA) was poor when compared with children who were older than $10 \mathrm{y}$, despite the presence of similar levels of IgG antibodies and a similar IgG isotype distribution (3). Pollard et al. (4) showed that differences in antibody avidity in infants are responsible for this striking observation. These authors reported that the avidity of antibodies against meningococcal reference strain H44/76, which expresses PorA serosubtype P1.7,16, was significantly lower in children who were younger than $1 \mathrm{y}$ than in those who were older than $10 \mathrm{y}$. However, the PorA specificity of the antibodies was not taken into consideration in this study. Therefore, differences in avidity maturation may depend on differences in PorA serosubtype of the strains that cause disease in these children. 
Candidate vaccines against serogroup B infections have not yet provided high efficacy rates in young children and infants (5-7). The search for a successful, widely protective meningococcal vaccine therefore still continues. The sector Vaccines of the National Institute of Public Health and Environment (RIVM; currently Netherlands Vaccine Institute) developed two candidate outer membrane vesicle (OMV) vaccines, mainly containing PorA as an antigen. A monovalent vaccine expresses PorA subtype P1.7-2,4 whereas a hexavalent vaccine contains six highly prevalent serosubtypes. Immunization of children with these vaccines induced a PorA-specific antibody response with a similar $\mathrm{IgG}$ isotype distribution as is seen after invasive meningococcal disease $(8,9)$. We and others also showed that vaccination with the monovalent OMV vaccine as well as the hexavalent vaccine induces a significant antibody avidity maturation $(9,10)$, which is a marker of the induction of immunologic memory (11-13).

The aim of the present study was to characterize the immune response of children after meningococcal septic shock with respect to SBA, IgG isotype, and antibody avidity. Furthermore, we determined whether the differences in avidity of anti-OMV antibodies were PorA specific. To this purpose, we selected acute- and convalescent-phase sera from children who all were infected with $N$. meningitidis serosubtype P1.7-2,4. Antibody avidity against the P1.7-2,4 antigen was measured and compared with the response against the heterologous antigen P1.7,16 and a PorA-deficient strain. In addition, we compared the avidity of antibodies to P1.7-2,4 OMV after convalescing from meningococcal infection with a serosubtype $\mathrm{P} 1.7-2,4$ strain and after vaccination with a P1.7-2,4 OMV vaccine.

\section{METHODS}

Patients. Acute-phase sera of children with meningococcal septic shock were drawn within $6 \mathrm{~h}$ of admission to the pediatric intensive care unit of the Sophia Children's Hospital, whereas convalescent-phase serum samples were obtained 1-3 mo after admission to the pediatric intensive care unit. The study was approved by the Medical Ethics Committee of the Erasmus MC. Written informed consent was obtained from the parents of the children. Sera of children with a positive blood culture for $N$. meningitidis serosubtype P1.4 were selected. The variable regions 1 and 2 of the PorA gene of these isolates were sequenced by the Netherlands Reference Laboratory for Bacterial Meningitis (Academic Medical Center Amsterdam/RIVM) and showed that these isolates all had P1.7-2,4 as a variable region 1/variable region 2 combination.

Avidity ELISA. Antibody avidity was measured using thiocyanate as chaotropic agent (9). In short, Immulon 2 (Dynex Technologies, Inc. Chantilly, USA) 96-well plates were coated overnight with $100 \mu \mathrm{L} /$ well of a monovalent OMV suspension at a concentration of $4 \mu \mathrm{g} / \mathrm{mL}$. OMVs from strain $\mathrm{H} 44 / 76$ with serosubtype P1.7,16, as well as OMVs from its isogenic variants with serosubtype P1.7-2,4 and the PorA-negative isogenic mutant H1.5 were used as a coating. Sera were diluted 1:100 in PBS-0.1\% Tween 80. A 3-fold serial dilution of serum samples was made in duplicate so that the two halves of a plate contained identical serum dilutions. Plates were incubated for $90 \mathrm{~min}$ at $37^{\circ} \mathrm{C}$. As a positive control, a serum sample from a volunteer who had been vaccinated with the hexavalent OMV vaccine in a phase I trial and had a high antibody titer against P1.7-2,4 and P1.7,16 was included on every plate. After three washes, $100 \mu \mathrm{L}$ of a $1.5-\mathrm{M}$ sodium thiocyanate (NaSCN) solution dissolved in PBS was added to one half of each plate and plain PBS was added to the other half. After incubation for $15 \mathrm{~min}$ at room temperature, all wells were washed three times and incubated with rabbit anti-human IgG 1:5000 conjugated with horseradish peroxidase (Dako A/S, Glostrup, Denmark) for 90 min at $37^{\circ} \mathrm{C}$. Subsequently after washing, 3,3',5,5'-tetramethylbenzidine substrate was added, and the reaction was allowed to proceed for $10 \mathrm{~min}$ and was stopped by adding $100 \mu \mathrm{L}$ of $2 \mathrm{M} \mathrm{H}_{2} \mathrm{SO}_{4}$ per well. The absorbance at $450 \mathrm{~nm}$ was read using an EL312e Bio-Kinetics reader. IgG antibody titers were determined as the dilution yielding $50 \%$ of the maximal OD. Samples with antibody titers below the assay's detection limit were assigned a value of 50 . Titers that were obtained after treatment with $\mathrm{NaSCN}$ were called "avidity titers." An avidity index (AI) was expressed as the percentage of antibodies that remained bound at the antigen coat after treatment with sodium thiocyanate: $\mathrm{AI}=$ titer $(\mathrm{NaSCN}+) /$ titer $(\mathrm{NaSCN}-) \times 100$.

IgG isotype ELISA. IgG isotype distribution of the convalescent patient sera was measured by an OMV ELISA using isotype-specific conjugates as described elsewhere (8). Anti-human $\mathrm{IgG}_{1}, \mathrm{IgG}_{2}, \mathrm{IgG}_{3}$, and $\mathrm{IgG}_{4}$ antibodies conjugated with horseradish peroxidase were used from Sanquin (Amsterdam, the Netherlands). IgG isotype titers were measured as described for the avidity ELISA, and samples with antibody titers below the detection limit were assigned a value of 50 .

$\boldsymbol{S B A}$. SBA titers were assessed as described previously (14). Human plasma with no detectable SBA against any of the strains used in this study was used as an exogenous complement source. SBA titers were expressed as the reciprocal of the lowest serum dilution that yielded $\geq 90 \%$ killing after $60 \mathrm{~min}$ of incubation.

Statistical analysis. IgG titers, avidity titers, and AIs were log-transformed, and geometric mean titers (GMT), geometric mean avidity titers (GMAT) and geometric mean avidity indices (GMAI) with 95\% confidence intervals (CIs) were calculated. Differences in GMTs and GMATs against OMVs of meningococcal strains with different serosubtypes were analyzed by Wilcoxon signed rank tests. Differences in GMAIs were analyzed by paired sample $t$ tests.

\section{RESULTS}

Serum samples from 21 children were used in this study. All children had a positive blood culture for $N$. meningitidis, serosubtype P1.7-2,4. The mean age of the patients was $6.3 \mathrm{y}$ (SD: $4.8 \mathrm{y}$; range: $0.5-13 \mathrm{y}$ ). Figure 1 shows the GMT measured against OMVs of a meningococcal strain that contained the homologous P1.7-2,4 or the heterologous P1.7,16 as PorA antigen or a PorA-deficient strain to test PorA specificity in acute and convalescent serum samples. In the acute phase of disease, GMTs were very low against all OMVs. In convalescent sera, there was no significant difference in GMTs against P1.7-2,4 and P1.7,16 $(p=0.071)$. GMT against H1.5 was significantly higher compared with $\mathrm{P} 1.7,16$ but not when compared with P1.7-2,4 ( $p<0.001$ and $p=0.063$, respectively).

The GMAT against P1.7-2,4 was significantly higher compared with the GMAT against P1.7,16 ( $p=0.004$; Fig. 2). GMTs were too low for assessment of GMAI in the acute-

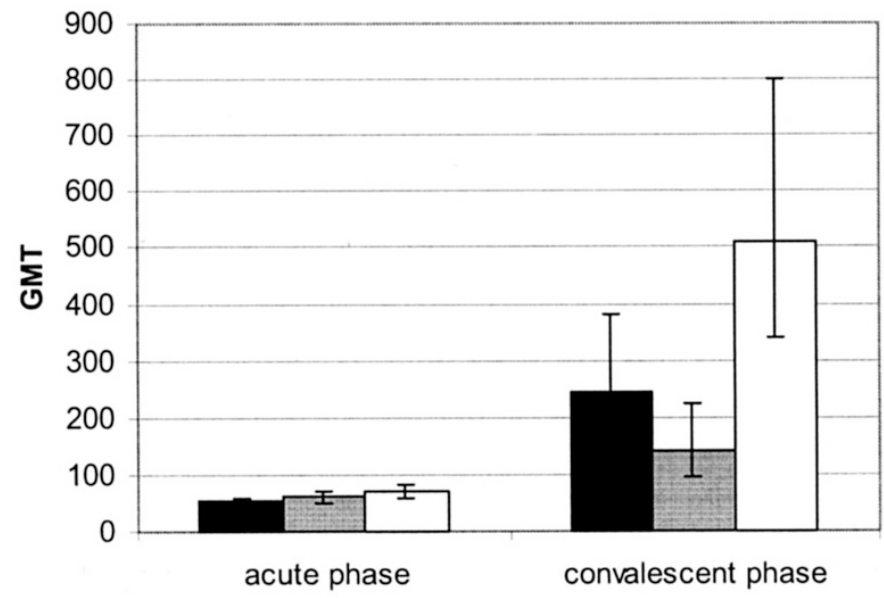

Figure 1. GMT of 21 sera in the acute phase and 1-3 mo after sepsis with $N$. meningitidis P1.7-2, 4 infection against OMVs from strain H44/76 either expressing P1.7-2, 4 or P1.7,16 PorA. Alternatively, the PorA-negative mutant H1.5 was used as source of antigen. $\square$ GMT 7-2,4; $\square$ GMT 7,16; $\square$ GMT H1.5. Error bars indicate 95\% CIs. 


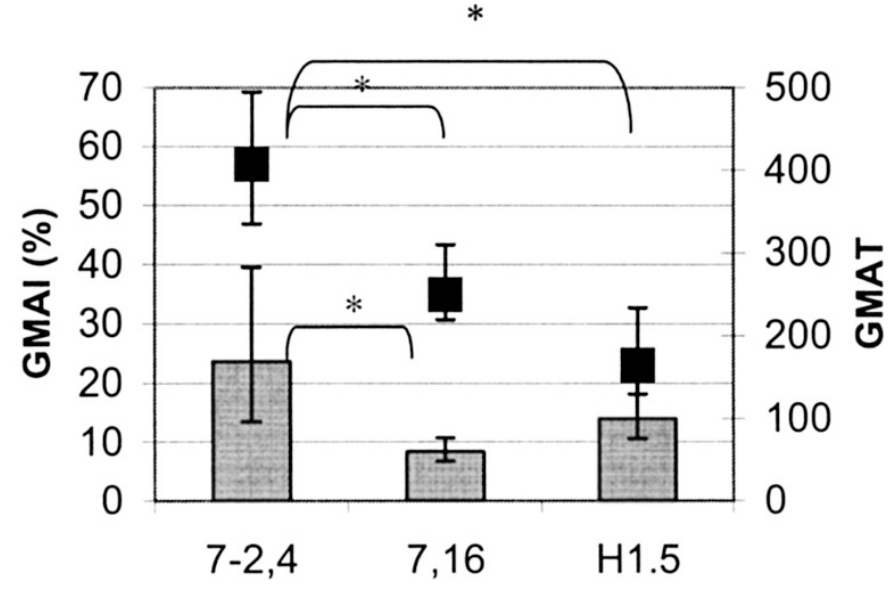

Figure 2. GMAI and GMAT in convalescent patient sera $(n=21) 1-3$ mo after sepsis with $N$. meningitidis serosubtype P1.7-2, 4. $\square$ GMAT; $\square$ GMAI. Error bars indicate $95 \%$ CIs. *Significant difference $(p<0.05)$

phase sera. However, in convalescent sera, the GMAI against P1.7-2,4 was 57\%, which is significantly higher than the GMAI against P1.7,16 (35\%) and H1.5 (23\%; $p=0.005$ and $p<0.001$, respectively). The IgG isotype distribution of the convalescent sera was dominated by $\operatorname{IgG}_{1}$ (GMT: 158; 95\% CI: 71-350), followed by $\operatorname{IgG}_{3}$ (GMT: 130; 95\% CI: 68-247), whereas no $\mathrm{IgG}_{2}$ or $\mathrm{IgG}_{4}$ was found. IgG titers in the acutephase sera were too low to assess $\mathrm{IgG}$ isotype distribution. SBA against strain H44/76 P1.7-2,4 did not reach titers $>2$ in any of the convalescent sera or against strains H44/76 P1.7,16 or H1.5.

\section{DISCUSSION}

In this study, we found that the high-avidity antibodies induced by a meningococcal infection in young children are specifically directed to the homologous PorA subtype of the disease-causing strain. Antibody avidity against OMVs of a strain with a heterologous PorA subtype is significantly lower, and antibody avidity against OMVs of a PorA-negative mutant strain is even lower. These results indicate that a specific immune response against PorA is generated as a result of invasive infection. Because antibody GMTs in the acute sera were so low, GMAI could not be measured in these samples. Therefore, we were unable to compare GMAI of acute-phase antibodies with GMAI of convalescent-phase antibodies and to assess whether avidity maturation occurred.

It is widely known that invasive meningococcal disease induces an antibody response mainly against PorA but also against PorB, LPS, and class 5 proteins. In this study, we focused on studying strain-specific PorA antibodies, because these have been found to be most relevant for bactericidal activity and protection from disease. Well-defined OMV suspensions were used as a coating in the avidity ELISA to measure antibody titers and the AI, in which PorA is the far most abundant antigen. These OMVs mainly contain either $\mathrm{P} 1.7-2,4$ or $\mathrm{P} 1.7,16$ PorA as a protein content, are depleted from PorB, and contain only low amounts of LPS and class 5 proteins. Therefore, avidity of antibodies against other antigens than PorA is not measured accurately in this set-up. However, the PorA-negative H1.5 OMVs contain large amounts of PorB. Earlier studies showed specific anti-PorB antibodies in convalescent-phase sera regardless of the serotype of the infecting strain (15). In our study, GMAI of antibodies against H1.5 OMVs were of low avidity.

We did not detect any significant SBA in the convalescent patient sera and therefore could not correlate SBA titers to GMAI. It was shown previously that SBA is low after a meningococcal infection, especially in young children $(1,3)$. Our group of patients had a mean age of $6.3 \mathrm{y}$, with only three children older than $10 \mathrm{y}$ and seven younger than $2 \mathrm{y}$, which may explain the absence of SBA in the convalescent sera. It should also be noted that we used human complement to measure bactericidal titers. In general, human complement has been shown to measure lower SBA titers compared with baby rabbit complement (16). Furthermore, maybe specific IgG titers were too low to reach the threshold level for SBA.

Recurrent infections by meningococci are exceptional except in complement-deficient individuals. This means that the immune response after invasive disease in otherwise healthy humans is protective, despite the absence of SBA in convalescent patient sera. Other mechanisms therefore may be responsible for induced protection after infection. Recently, several studies have shown protection in infant rat models against meningococcal infection in absence of SBA activity $(17,18)$. The most likely mechanism responsible for protective activity in the absence of SBA is opsonization. Opsonic activity was found in patients after meningococcal serogroup B infection (19). Also, animal studies have shown that protective activity was associated with high-avidity antibodies $(18,20,21)$. The PorA-specific avidity induced after infection therefore may play an import role in protection.

Although important differences can be expected between the immune responses of healthy toddlers who are vaccinated with a meningococcal OMV vaccine and children who are recovering from meningococcal septic shock, we tried to compare the characteristics of the immune response in these patients with the immune response seen in children who are immunized with a monovalent P1.7-2,4 OMV vaccine. The IgG isotype distribution was similar between the two groups (9). The GMAI in convalescent sera is lower in comparison with antibody avidity induced by the monovalent $\mathrm{P} 1.7-2,4 \mathrm{OMV}$ vaccine: 57 versus $73 \%$. This may suggest that vaccination with this OMV vaccine induces a better immune response than invasive meningococcal disease and provides an encouraging view on the protection against meningococcal disease offered by this vaccine.

Acknowledgments. We thank René Kornelisse and Ester de Kleijn for collecting patient serum samples and Wendy Keijzers for sequencing part of the PorA gene of the patient isolates.

\section{REFERENCES}

1. Wedege E, Hoiby EA, Rosenqvist E, Bjune G 1998 Immune responses against major outer membrane antigens of Neisseria meningitidis in vaccinees and controls who contracted meningococcal disease during the Norwegian serogroup B protection trial. Infect Immun 66:3223-3231

2. Wedege E, Bolstad K, Wetzler LM, Guttormsen H 2000 IgG antibody levels to meningococcal porins in patient sera: comparison of immunoblotting and ELISA measurements. J Immunol Methods 244:9-15 
3. Pollard AJ, Galassini R, van der Voort EM, Booy R, Langford P, Nadel S, Ison C, Kroll JS, Poolman J, Levin M 1999 Humoral immune responses to Neisseria meningitidis in children. Infect Immun 67:2441-2451

4. Pollard AJ, Levin M 2000 Production of low-avidity antibody by infants after infection with serogroup B meningococci. Lancet 356:2065-2066

5. Bjune G, Hoiby EA, Gronnesby JK, Arnesen O, Fredriksen JH, Halstensen A, Holten E, Lindbak AK, Nokleby H, Rosenqvist E, et al. 1991 Effect of outer membrane vesicle vaccine against group B meningococcal disease in Norway. Lancet 338:10931096

6. Boslego J, Garcia J, Cruz C, Zollinger W, Brandt B, Ruiz S, Martinez M, Arthur J, Underwood P, Silva W, Moran E, Hankins W, Gilly J, Mays J 1995 Efficacy, safety, and immunogenicity of a meningococcal group B (15:P1.3) outer membrane protein vaccine in Iquique, Chile. Chilean National Committee for Meningococcal Disease. Vaccine 13:821-829

7. de Moraes JC, Perkins BA, Camargo MC, Hidalgo NT, Barbosa HA, Sacchi CT, Landgraf IM, Gattas VL, Vasconcelos Hde G, Gral IM, et al. 1992 Protective efficacy of a serogroup B meningococcal vaccine in Sao Paulo, Brazil. Lancet 340:1074-1078

8. de Kleijn E, van Eijndhoven L, Vermont C, Kuipers B, van Dijken H, Rumke H, de Groot R, van Alphen L, van den Dobbelsteen G 2001 Serum bactericidal activity and isotype distribution of antibodies in toddlers and schoolchildren after vaccination with RIVM hexavalent PorA vesicle vaccine. Vaccine 20:352-358

9. Vermont CL, van Dijken HH, van Limpt CJ, de Groot R, van Alphen L, van Den Dobbelsteen GP 2002 Antibody avidity and immunoglobulin G isotype distribution following immunization with a monovalent meningococcal B outer membrane vesicle vaccine. Infect Immun 70:584-590

10. Longworth E, Borrow R, Goldblatt D, Balmer P, Dawson M, Andrews N, Miller E, Cartwright K 2002 Avidity maturation following vaccination with a meningococcal recombinant hexavalent PorA OMV vaccine in UK infants. Vaccine 20:2592-2596

11. Goldblatt D, Borrow R, Miller E 2002 Natural and vaccine-induced immunity and immunologic memory to Neisseria meningitidis serogroup $\mathrm{C}$ in young adults. J Infect Dis $185: 397-400$

12. Joseph H, Miller E, Dawson M, Andrews N, Feavers I, Borrow R 2001 Meningococcal serogroup A avidity indices as a surrogate marker of priming for the induction of immunologic memory after vaccination with a meningococcal $\mathrm{A} / \mathrm{C}$ conjugate vaccine in infants in the United Kingdom. J Infect Dis 184:661-662

13. Richmond P, Borrow R, Goldblatt D, Findlow J, Martin S, Morris R, Cartwright $\mathrm{K}$, Miller E 2001 Ability of 3 different meningococcal C conjugate vaccines to induce immunologic memory after a single dose in UK toddlers. J Infect Dis 183:160-163

14. van der Voort ER, van der Ley P, van der Biezen J, George S, Tunnela O, van Dijken H, Kuipers B, Poolman J 1996 Specificity of human bactericidal antibodies against PorA P1.7,16 induced with a hexavalent meningococcal outer membrane vesicle vaccine. Infect Immun 64:2745-2751

15. Guttormsen HK, Wetzler LM, Naess A 1993 Humoral immune response to the class 3 outer membrane protein during the course of meningococcal disease. Infect Immun 61:4734-4742

16. Santos GF, Deck RR, Donnelly J, Blackwelder W, Granoff DM 2001 Importance of complement source in measuring meningococcal bactericidal titers. Clin Diagn Lab Immunol 8:616-623

17. Welsch JA, Moe GR, Rossi R, Adu-Bobie J, Rappuoli R, Granoff DM 2003 Antibody to genome-derived neisserial antigen 2132 , a Neisseria meningitidis candidate vaccine, confers protection against bacteremia in the absence of complement-mediated bactericidal activity. J Infect Dis 188:1730-1740

18. Welsch JA, Granoff DM 2004 Naturally acquired passive protective activity against Neisseria meningitidis Group $\mathrm{C}$ in the absence of serum bactericidal activity. Infect Immun 72:5903-5909

19. Halstensen A, Lehmann AK, Guttormsen HK, Vollset SE, Bjune G, Naess A 1991 Serum opsonins to serogroup B meningococci after disease and vaccination. NIPH Ann 14:157-165, discussion 166-167

20. Usinger WR, Lucas AH 1999 Avidity as a determinant of the protective efficacy of human antibodies to pneumococcal capsular polysaccharides. Infect Immun 67:23662370

21. Lucas AH, Granoff DM 1995 Functional differences in idiotypically defined IgG1 anti-polysaccharide antibodies elicited by vaccination with Haemophilus influenzae type B polysaccharide-protein conjugates. J Immunol 154:4195-4202 